greater than 0.5 and predicts that 3000 stars brighter than the I8th magnitude will show similar motions, about one star per Io cubic parsecs.

Dr Vyssotsky reported plans for an extension of the proper motion programme at the McCormick Observatory. Photographs of their early fields will be taken after an interval of twenty-four years and among other values the tau-components for about 20,000 stars will be derived.

Dr Slocum called attention to the fact that for a number of parallax stars the residuals seem to be periodic and urged that in such cases the series of plates be extended over enough epochs to verify or disprove the apparent periodicity.

Dr Schilt gave a report of progress in his investigations of the probable errors of trigonometric parallaxes. He found that the probable error of a plate of unit weight increased with the distance of the comparison stars from the parallax stars. It was suggested that the errors might arise from solar or lunar tides in our atmosphere.

In connection with the determination of the magnitudes of the parallax stars and comparison stars the need of more standard magnitude series scattered over the whole sky was stressed.

In the discussion of spectroscopic parallaxes Dr Harper noted that the differences between these and trigonometric parallaxes are often far greater than their probable errors. A list of stars showing great differences should be published.

Dr Cecchini gave a report of progress at Milan upon the problem of deriving spectroscopic parallaxes of I200 stars of spectral classes B, A and F. Microphotometric curves for six thousand spectra have been made and characteristic curves for all plates have been derived. He also reported on the determination of trigonometric parallaxes.

\title{
COMMISSION 25 (STELLAR PHOTOMETRY)
}

Acting President: Dr W. Baade.

SeCretary: Prof. H. L. VANDERLinden.

Two meetings of the Commission were held.

The following topics were mainly discussed:

(I) Zero point of photographic magnitude and colour index. The President recalled the history of determining the zero point of the international photographic scale and the system of colours. This was done in such a way that the colour index should be zero for Ao stars between the magnitudes 5.5 and 6.5 . The magnitude scale of the N.P.S. is in harmony with this decision. But a deviation of $+0^{\mathrm{m}} \cdot 04$ for Ao stars has been observed now, due mainly to the fact of the small number of Ao stars between those magnitudes.

The Polar Sequence however is not representative for the whole sky because of the existence of a colour excess of +o.1o mag. But Drs Baade and Shapley suggested that no decision should be taken now and that the values of I922 should still be used.

Mrs Payne Gaposchkin's suggestion (p. 2r8) was adopted by 7 against 2 votes. The proposal of Prof. van Rhijn, supported by Prof. Schilt, to define the zero point of the international sequence by magnitudes of stars 2,4 and 5 of the N.P.S. was discussed. It was pointed out, however, that the zero point of the scale could not be defined by such a small number of stars (see above). 
(2) Aluminized mirrors. Dr Pettit has computed the colour equation which is to be expected for an aluminized mirror under the assumption of black-body radiation for the stars. A direct determination of this colour equation at Mt Wilson shows that it is of the order of $0^{\mathrm{m}} \cdot 3$ for early B stars, of the order of $\mathrm{o}^{\mathrm{m}} \cdot \mathrm{I}$ for A stars and negligible for Go stars. This correction can thus be neglected in our galactic system because most of the B-type stars are reddened. But for faint stars in extra-galactic systems differences occur, and screens should be used in order to make the results comparable. Aluminized mirrors are very sensitive to humidity, so that their use will, in general, be restricted to a few places in dry climates. Many improvements will undoubtedly be made in the future, so that it is advisable, according to Dr Shapley and Prof. Hertzsprung, to take up discussion again later and not to take any decision at this time.

One may meanwhile proceed in such a way that the colour interval of effective wave-lengths, say 200 A., varies the least possible between the spectral types A and $K$.

(3) The colour excess should be determined in the N.P.S. with respect to nonobscured stars in high galactic latitudes. A list of non-obscured A-type stars should be made up. Dr Malmquist has already started spectrophotometric work in a region of 70 square degrees around the pole.

From a discussion of the distribution of faint stars in a region of $10^{\circ}$ around the pole, Dr Shapley has been able to estimate the general absorption, corresponding with a colour excess of $\mathrm{O}^{\mathrm{m} \cdot \mathrm{r}}$.

Prof. Stebbins, on the other hand, has made comparisons with the photo-electric cell, from which it results that a colour excess is appearing from the 8 th magnitude on.

(I) At the second meeting, Prof. Hertzsprung presented a memorandum to the Commission. In connection with this the following resolution was adopted:

"Commission 25 on Stellar Photometry proposes the appointment of a subcommission which should consider the redefinition of standard magnitudes." (See Prof. Hertzsprung's memorandum.) This memorandum will figure in the report of the Commission, to be published in the Transactions of the Union. The following astronomers were proposed as members of this sub-commission: F. H. Seares (President), W. Baade, B. Lindblad, E. Hertzsprung, H. Shapley, W. R. Green, J. Hellerich.

\section{Memorandum by Prof. E. Hertzsprung}

With much interest $I$ have read the discussion, as printed in the Draft Report of Commission 25, concerning the question of the zero point of photographic magnitude.

It may well be that the proposal to take the magnitudes of the N.P.S. as a standard will reasonably respond to the wants of the present day. But I expect that before long we will need a more precise definition of the point in question and I am, therefore, of opinion that already at present we ought to face the extension of this problem.

The procedure is to select a series of stars distributed over the whole range of magnitude required and containing for each magnitude stars of different colour. A systematic change of the average colour with magnitude should be avoided as far as possible. For each star selected in this way accurate magnitudes are determined, corresponding to two different and fixed values of $\lambda_{\text {eff }}$.

The N.P.S. is not up to these claims. The variation of colour within each magnitude is rather small and there is a marked systematic change of the average colour 
with magnitude. No faint white stars are found in the N.P.S. Of the two sorts of magnitudes, $m_{\mathrm{pg}}$ and $m_{\mathrm{pv}}$, as determined for the stars of the N.P.S., only $m_{\mathrm{pv}}$ has practically the same $\lambda_{\text {eff }}$ for ordinary stars of all colours, while $m_{\mathrm{pg}}$ does not satisfy this condition, which is fundamental for accurate photometry.

I therefore consider as the first thing to be done the determination of new practically monochromatic magnitudes corresponding to a $\lambda_{\text {eff }}$ sufficiently different from that of $m_{\mathrm{pv}}$, which may be taken as approximately ${ }^{\mu .} 55$. The new $\lambda_{\text {eff }}$ should be rather short in order to give strong colour indices by combination with $m_{\mathrm{pv}}=m_{550}$. For this purpose I propose spectral photometry of the region between $\mathrm{H}_{\delta}$ and $\mathrm{H}_{\epsilon}$, which can be easily picked out on nearly all spectra of very small dispersion. The corresponding $\lambda_{\text {eff }}$ is about $\mu_{4} 403$. The colour indices $m_{403}-m_{550}$ show something like double the range of the ordinary colour indices $m_{\mathrm{pg}}-m_{\mathrm{pv}}$.

The connection of another system of magnitudes $m_{\mathrm{s}}$ with those of the standard region is then established in the following way. The three unknowns to be determined are: the difference in zero point, the proportion between the scales and the $\lambda_{\text {eff }}$. The equations of condition then take the form:

$$
m_{\mathrm{s}}=A+B\left(m_{403}+m_{550}\right)+C\left(m_{403}-m_{550}\right),
$$

where $m_{403}+m_{550}$ and $m_{403}-m_{550}$ are, as they ought to be, independent of each other. (This is not the case with $m_{\mathrm{pg}}$ and $m_{\mathrm{pg}}-m_{\mathrm{pv}}$ sometimes incorrectly used in the equations of condition.)

The least squares solution gives the constants $A, B$ and $C$. If a sufficient number of stars is used reliable values of their mean errors may also be derived.

The place of the colour conception of $m_{\mathrm{s}}$ between $m_{903}$ and $m_{550}$ is then determined by:

$$
\frac{B+C}{2 B} m_{403}+\frac{B-C}{2 B} m_{650} \text {. }
$$

For the reasons mentioned above the N.P.S. is not well suited for a good separation of the coefficients $B$ and $C$ or, in other words, of the equations of magnitude and of colour. Probably a better sequence, containing also faint white stars, could be found nearer the Milky Way. On the other hand the possibility of using "white dwarfs" photometrically for the same purpose should be kept in mind.

Ordinary photographic magnitudes are, owing to the comparatively wide range of $\lambda^{-4}$ acting on the plate, inconvenient for accurate photometry as soon as stars of different colour and images of systematically different intensity are involved.

In the case of $m_{\mathrm{pg}}$ the $\lambda_{\text {eff }}$ not only varies considerably with the colour of the stars, but the faintest images, which are the only ones available for the faintest accessible stars, show a markedly shorter $\lambda_{\text {eff }}$ than the well exposed images, which are preferable from the point of view of accuracy, and therefore used for the brighter stars.

As a consequence of these facts the ordinary photographic magnitudes cannot be satisfactorily represented by the simple "monochromatic" formula given above. In order to make this possible the addition of terms of higher order or separate treatment of stars of different colour classes is wanted.

For the time being we will certainly be forced to make further use of "photographic" magnitudes in practice, but they should be abandoned as standards as soon as possible and replaced by another system of practically monochromatic magnitudes to be used in combination with $m_{\mathrm{pv}}$.

(2) The following resolution, which was already endorsed by Commissions 28 on Nebulae and Clusters and 33 on Stellar Statistics, was adopted by Commission 25: 
"Commission 25 on Stellar Photometry proposes the appointment of a subcommission on magnitude sequences, noting that for work on stellar distribution, variable stars, external galaxies, faint asteroids and faint proper motion stars the need of precise photographic and visual magnitudes is very great."

The proposed members of this sub-commission are: Mrs Payne Gaposchkin (President), A. N. Vyssotsky, A. Wallenquist. There will be further possibility of co-option.

(3) Prof. Hertzsprung made the following recommendation concerning the publication of photometric catalogues:

"In two recent publications of photometric catalogues the new results are not given separately, but have been presented only in combination with earlier determinations made by others. I therefore want to emphasize the necessity of publishing original results independently in order to enable anyone to combine the different series available in the way he wishes."

(4) $\operatorname{Dr} \AA$. Wallenquist presented the following suggestions concerning accurate photometric standard regions in the southern sky:

"It will not be necessary here to point out the very great importance of having a number of accurate photometric standard regions well distributed over the sky. The extensive programme of making standard regions proposed by Dr Shapley seems to me, however, to involve such a tremendous work, that its completion within a reasonable time is impossible. We need, however, as soon as possible, a number of sequences of accurate magnitudes. Therefore, I should like to propose, not in the first place to increase the number of sequences, but to extend and improve the sequences which are available at the present moment. For northern observatories the N.P.S. is available but for observatories situated in the southern hemisphere the lack of good standards makes good photometric work difficult. In Harvard Annals, 85, Miss Henrietta S. Leavitt gives a great number of standards of magnitudes for the Astrographic Catalogues. In the southern hemisphere the Harvard sequences of standard stars were well distributed over the zones $-2^{\circ} \cdot 5,-9^{\circ} \cdot 5,-16^{\circ} \cdot 5,-23^{\circ} \cdot 5,-31^{\circ} \cdot 5,-40^{\circ} \cdot 5,-51^{\circ} \cdot 5,-64^{\circ} \cdot 5$. The limiting magnitudes of the sequence stars are about 14.0 . The present magnitudes are perhaps not of a very high accuracy, but they will form a good basis for further extensions. My proposal is to redetermine the magnitudes of the stars in the regions mentioned, to extend the number of stars to fainter magnitudes, to determine accurate photo-visual and photo-red magnitudes and to try to bring all the magnitudes to the same system as the one of the present N.P.S."

(5) Prof. Hagihara had communicated to the President an account of some photometric projects in Japan. It is intended to use a 26 -inch refractor of Zeiss at the Tokyo Observatory at Mitaka for photographic photometry. The Director, Dr Sekiguti, would welcome suggestions with regard to the work to be undertaken. Mr Oikawa of the Tokyo Observatory is at present making a photometric study of some stars with a Brashear I2-inch camera. 